\title{
DESIGUALDAD SALARIAL Y CAMBIO TECNOLÓGICO en la Frontera Norte de MÉXico
}

\author{
Luis Huesca y Gloria Ochoa ${ }^{1}$
}

Fecha de recepción: 17 de febrero de 2016. Fecha de aceptación: 15 de agosto de 2016.

\begin{abstract}
RESUMEN
El artículo explica el comportamiento de la desigualdad salarial de México y su región correspondiente a la Frontera Norte durante el periodo 2005-2012. Se empleó un índice tradicional y de fácil interpretación, el coeficiente de Gini, mismo que es descompuesto de manera exacta mediante un enfoque de juegos cooperativos y mediante el cual se obtienen las causas de su gradual reducción misma que pasó de ser de 0.435 a 0.400 para el año 2012. Los resultados muestran que es al interior de los grupos -en un 70\%que ha permitido reducir las distancias de los salarios por tipo de tareas en el puesto de trabajo para la frontera y por habilidades para el país.

Palabras clave: desigualdad salarial, mercado laboral, coeficiente de Gini, cambio tecnológico, Frontera Norte.
\end{abstract}

Clasificación JEL: J08, J31, O18, O33.

\section{Wage Inequality and Technological Change ALONG THE Northern Border OF MeXico}

\begin{abstract}
This paper explains the behavior of wage inequality in Mexico and the corresponding region along the northern border in the time period 2005-2012. We use a traditional and easy-to-interpret coefficient, the Gini index, which is accurately decomposed using a cooperative game approach, to obtain the causes behind its gradual decline, as the index fell from 0.435 to 0.400 by 2012 . The results show that it is within groups - by $70 \%$ - that the wage gap has been reduced by type of task in the job position for the border region and by skills for the country.

Key Words: Wage inequality, labor market, Gini index, technological change, northern border.

${ }^{1}$ Centro de Investigación en Alimentación y Desarrollo, A.C., México, y Facultad de Economía Internacional-Universidad Autónoma de Chihuahua, México, respectivamente. Correos electrónicos: lhuesca@ciad.mx y gochoa@uach.mx, respectivamente.
\end{abstract}




\section{INÉGALITÉ SALARIALE ET CHANGEMENT TECHNOLOGIQUE À LA FRONTIÈRE NORD DU MEXIQUE \\ Résumé}

L'article explique l'évolution de l'inégalité salariale du Mexique et de sa région correspondant à la frontière nord au cours de la période 2005-2012. Il y est employé un indice traditionnel et facile à interpréter du coefficient Gini, lequel est décomposé de manière exacte en adoptant une perspective de jeux coopératifs à travers laquelle on parvient aux causes de sa progressive réduction, puisqu'il est passé de 0.435 à 0.400 pour l'année 2012. Les résultats montrent que c'est au sein des groupes - à hauteur de $70 \%$ - qu'il a permis de réduire les écarts de salaires pour le type de tâche sur le poste de travail en ce qui concerne la frontière, et selon les habiletés dans le pays.

Mots clés: inégalité salariale, marché du travail, coefficient de Gini, changement technologique, frontière nord.

\section{DESIGUALDADE SALARIAL E MUDANÇA TECNOLÓGICA NA FRONTEIRA NORTE DO MÉXICO}

\section{Resumo}

O artigo explica o comportamento da desigualdade salarial do México e sua regiáo que corresponde à Fronteira Norte durante o período de 2005-2012. Para tanto, se usou um índice tradicional e de fácil interpretação como o de Gini, mesmo que é decomposto de maneira exata através de um enfoque de jogos cooperativos e no qual se encontram as causas da sua gradual redução, que passou de 0.435 a 0.400 para o ano de 2012. Os resultados mostram que é no interior dos grupos - num $70 \%$ - que permitiu reduzir as distâncias dos salários por tipo de tarefas no posto de trabalho, para a fronteira, e por habilidades, para o país.

Palavras-chave: desigualdade salarial, mercado laboral, coeficiente de Gini, câmbio tecnológico, fronteira norte.

\section{墨西哥北部边境工资不平等及技术转变}

摘要

本文分析墨西哥北部边境地区2005-2012年间的工资不平等现象。我们 使用传统的易于阐释的基尼系数, 通过合作博亦指数准确分解来得到这 一系数2012 年从 0.435 逐渐下降到 0.400 的原因。分析结果显示就北部 边境地区而言，在不同工作类型中可因工所任务的差别而缩小工资差距 (占70\%)，就全国而言，可因工作熟练程度的不同而缩小工资差距。 关键词：工资不平等，劳动力市场，基尼系数，技术变革，北部边境 


\section{INTRODUCCIÓN}

La reciente etapa de la economía mundial se caracteriza por tener un sector económico que gira en torno a las tecnologías de la información con una serie de políticas orientadas a la competencia en el mercado internacional. En las últimas décadas el proceso de cambio tecnológico ha provocado una tendencia hacia la contratación de individuos con mayores capacidades y habilidades mediante los cuales, las empresas buscan mayor rentabilidad y así como eficiencia en su producción.

Cabe recordar que desde la Revolución Industrial se ha sustituido la mano de obra por maquinaria; recientemente la constante competencia internacional ha provocado que las empresas adquieran nuevas herramientas para hacer más eficientes sus procesos productivos, y debido a que dicha tecnología requiere de habilidades específicas, por parte de los trabajadores, la sustitución es un hecho evidente.

El análisis sobre el desarrollo tecnológico y su efecto en el mercado de trabajo, sugiere que al existir una mayor productividad derivada del uso de innovaciones tecnológicas, se generará una demanda de trabajadores con más habilidades (individuos calificados) que a su vez originarán mayores remuneraciones salariales. Dicho progreso tecnológico se analiza recientemente basándose en las habilidades de los trabajadores. De acuerdo con Acemoglu y Autor (2011) una manera de medir el rendimiento de la mano de obra es a través de observar el salario relativo de los individuos calificados y no calificados, con este análisis se ha observado un sesgo debido al uso de tecnología en favor del trabajo calificado y en detrimento del menos calificado lo que conlleva a una desigualdad salarial.

Recientemente, una nueva literatura argumenta que los cambios en la asignación de los lugares de trabajo o "tareas" entre el capital y el trabajo, o entre trabajadores nacionales y extranjeros, han alterado la estructura de la demanda laboral en los países industrializados y se ha fomentado la polarización del empleo. De acuerdo con Autor (2013) el "enfoque de tareas" aplicado a los mercados laborales ofrece un prometedor ajuste de herramientas conceptual para el estudio de las fuerzas de éste.

Bajo este marco de análisis se desarrolla el presente trabajo, mismo que tiene como objetivo analizar el comportamiento que ha tenido la desigualdad salarial entre distintos grupos de ocupaciones en la región de la Frontera Norte de México (FNM) durante el periodo 2005-2012. Para tal efecto, el documento está estructurado de la siguiente manera: en primer lugar, se presenta una revisión de literatura en relación con el cambio tecnológico en el mercado laboral 
y la desigualdad salarial derivada de éste, además se agrega una breve descripción de la problemática del mercado laboral mexicano. En segundo lugar, se plantea la situación del mercado de trabajo en la FNM y la desigualdad salarial en esta región explicada por el cambio tecnológico. En tercer lugar, se presenta la metodología correspondiente a la descomposición exacta del índice de Gini para medir la desigualdad, seguida de los resultados obtenidos. Por último, se muestran las conclusiones pertinentes.

\section{CAMBIO TECNOLÓGICO, MERCADO LABORAL Y DESIGUALDAD SALARIAL}

De acuerdo con la Hipótesis del Cambio Tecnológico Sesgado (нстs), tras la introducción de tecnología en el mercado laboral se produce un incremento en la demanda de empleados con más habilidades, lo que provoca un sesgo en favor de los trabajadores calificados y en detrimento de los no calificados, provocando un incremento en la desigualdad salarial. Dicha hipótesis aproxima el cambio tecnológico mediante el uso de equipo de cómputo, debido principalmente a la expansión de esta herramienta en los centros de trabajo.

Bajo el enfoque de la HCTs, una de las posibles respuestas al origen de la desigualdad salarial, es la referente a los cambios en la demanda, derivados del cambio tecnológico y el sesgo en favor de los trabajadores más calificados.

Este fenómeno ha sido probado para países desarrollados, por diversos investigadores a lo largo de las décadas (Autor, Katz y Kearney, 2008; Acemoglu et al., 2011), diversas investigaciones aseguran que la introducción de tecnologías en el trabajo genera una demanda de trabajadores mayormente calificados, o bien, que las tareas que estos realizan, sean complementarias con estas tecnologías provocando un incremento en su salario. En este contexto, la educación resulta un elemento de gran importancia, debido a que de acuerdo con la escolaridad se determina el acceso al uso nuevas tecnologías.

Para países en desarrollo como México, se ha observado que tras la firmal del Tratado de Libre Comercio de América del Norte (TLCAN), se presentaron modificaciones en materia de inversión extranjera directa y el esquema maquilador llevado a cabo durante el proceso de restructuración económica permitió la entrada al país de empresas transnacionales que incorporaron más tecnología en los procesos productivos, provocando efectos en la demanda de mano de obra y en los salarios (Hanson, 2003).

Dicho cambios en la economía mexicana ha motivado diversas investigaciones para analizar los cambios en la estructura salarial y la composición del empleo, y una de las formas básicas que se ha implementado para estudiar el 
impacto del cambio tecnológico en el mercado laboral, es analizar los cambios en la mano de obra y las implicaciones salariales a las que se enfrentan los trabajadores de acuerdo a sus habilidades, es precisamente esta, la forma en la que se da explicación a los cambios en la desigualdad salarial en este trabajo de investigación.

\section{La desigualdad salarial en México}

Durante la década de los ochenta en México se presentó un incremento en la disparidad salarial, entre las principales causas que explicaron dicho aumento se encuentran las siguientes: 1) cambios en la demanda: globalización y mayor competencia de bienes intensivos en mano de obra, cambio tecnológico y demanda sesgada en favor de mano de obra especializada; 2) cambios en la oferta: creciente participación femenina en el mercado laboral y aumento de la oferta laboral de jóvenes; 3) cambios institucionales del mercado laboral: desregulación del salario mínimo y el descenso de la participación sindical (Castro y Huesca, 2007).

Por su parte, Esquivel y Rodríguez (2003) analizaron el periodo previo y posterior al TLCAN, y demostraron que a lo largo del periodo 1988-2000, la brecha entre salarios de trabajadores calificados y no calificados en México se incrementó en alrededor del $27 \%$.

Posteriormente, Campos, Esquivel y Lustig (2014), estudiaron la desigualdad salarial en México durante 1989-2010, encontrando que presentó dos comportamientos distintos durante este periodo. El primero se dio durante 1989 y hasta mediados de 1990 con un incremento del coeficiente de Gini de 0.548 a 0.571 . El segundo cambio se daría de mediados de los años noventa hasta el 2010, mostrándose una reducción del coeficiente de Gini a 0.510. Los autores aseguran que una de las posibles causas en la reducción de la desigualdad salarial fue debido a una sobreoferta de trabajadores calificados.

Por su parte, Lustig, López y Ortiz (2013) abordan la desigualdad en América Latina y los casos específicos de México, Argentina y Brasil. Para el caso de México, los autores examinan de forma específica la disparidad del ingreso laboral y observan una reducción de la misma, concluyendo que esta, se debió principalmente a la caía de la prima salarial de los trabajadores calificados que además se puede atribuir a los cambios en la composición de la oferta y demanda, así como factores institucionales.

En otro estudio realizado por Huesca y Rodríguez (2008), se probó que en México al inicio del año 2000 se presentó una estabilización de la desigualdad, acompañada de una reducción salarial para el grupo de trabajadores califica- 
dos. En este mismo estudio para el caso de la FNM se mostró un proceso de homogeneización en los salarios con mayor peso para el trabajo no calificado, evidencia que contrasta con la tendencia de nuevas técnicas de producción que pudieran utilizar de forma más intensiva trabajo calificado y que al parecer no ha ocurrido en ańos recientes.

De acuerdo con la evidencia presentada, la desigualdad salarial en México ha mostrado distintos patrones, mismos que se atribuyen a los cambios producidos en la oferta de trabajo y la posibilidad de un cambio tecnológico sesgado, en este sentido, resulta interesante analizar la descomposición de la desigualdad salarial, para conocer cada uno de los factores que contribuyen a dicha disparidad.

\section{El mercado laboral mexicano}

Como se mencionó anteriormente, la economía mexicana ha presentado modificaciones en su estructura productiva, y aun cuando se produjo un incremento de las oportunidades provenientes de un mayor flujo comercial, los indicadores de empleo de acuerdo con Mendoza (2010) han mostrado una incapacidad del mercado laboral de absorber la demanda de trabajo que se genera. Por ello, el empleo, la subocupación y la migración hacia Estados Unidos, se han presentado como parte relevante de las características del mercado laboral mexicano.

El mercado de trabajo de este país, muestra fenómenos recurrentes, tales como el incremento del empleo informal, el crecimiento de flujos de trabajadores mexicanos que emigran a Estados Unidos y un incremento en la dinámica de actividades industriales y manufactureras.

En este mismo sentido, Castro (2007) quien realizó un análisis de la brecha salarial de 1992 a 2002, afirma que el mercado de trabajo mexicano no ha generado los empleos requeridos para una oferta laboral que crece constantemente.

Por lo tanto, el caso de México resulta contradictorio a la teoría que asegura que a mayor nivel educativo se obtendrán mayores beneficios salariales, un ejemplo de esto, es el incremento de la matrícula de estudiantes universitarios, y a su vez el aumento en las tasas de desempleo de los grupos con más grados de escolaridad, cuya principal consecuencia es la ocupación de puestos de trabajo de baja especialización, informalidad y bajos salarios.

Otra evidencia que contribuye a esta explicación se muestra en la gráfica 1, donde se presentan los salarios para el periodo 2005-2012 de trabajadores calificados (más de 12 años de escolaridad) y no calificados (menos de 12 años de 
Gráfica 1. Salarios promedio por tipo de calificación en México 2005-2012

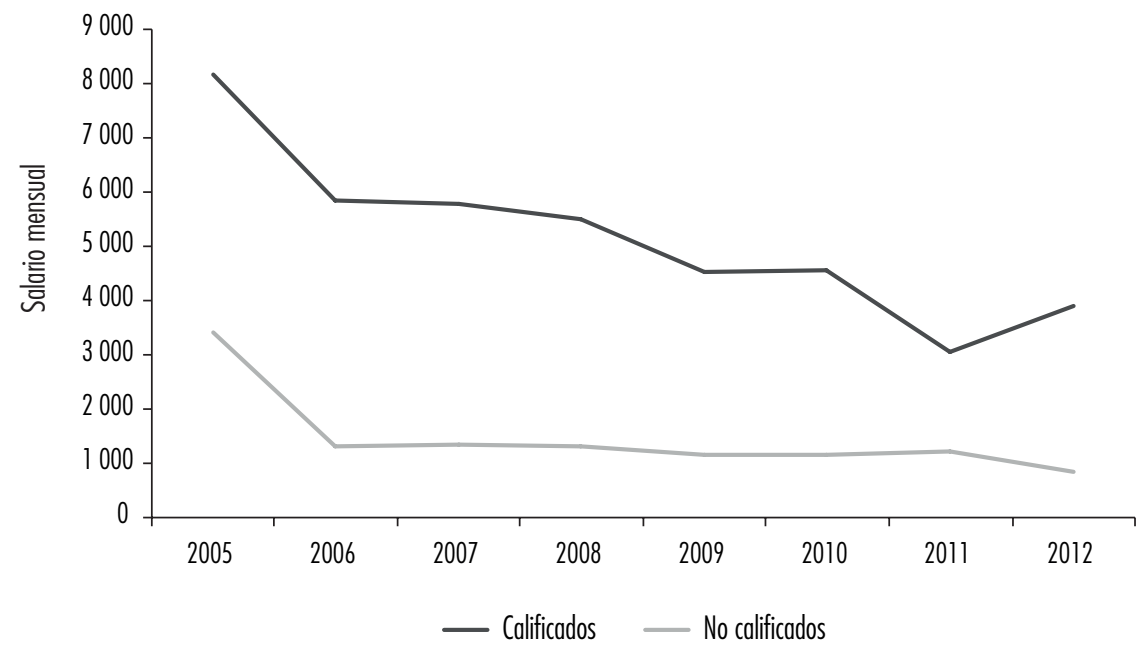

Fuente: elaboración propia con datos de ENOE.

escolaridad), en la que se observa una caída drástica de la retribución salarial media para el grupo de personas que cuentan con un mayor nivel educativo.

La evidencia expuesta anteriormente muestra como a lo largo de las últimas décadas, los desajustes en el mercado laboral mexicano han imposibilitado el desarrollo pleno de los trabajadores, aun cuando se han introducido procesos productivos más tecnificados vinculados a las destrezas con las que cuenten dichos empleados sus salarios no son retribuidos en función de alguna habilidad.

\section{EL CASO DE LA FRONTERA NORTE DE MÉXICO}

El estudio específico de la Frontera Norte de México (FNM), es fundamental para comprender la introducción de cambio tecnológico y sus implicaciones en materia salarial, debido a que es una de las regiones del país que se caracteriza por un desarrollo económico avanzado comparado con la Frontera Sur, e incluso con algunas ciudades del centro de la República mexicana. Ayala, Chapa y Hernández (2009) explican que los estados del norte tienen una vocación industrial debido a que la industria manufacturera determina la actividad económica regional; su dinamismo se produce por la cercanía con Estados Unidos, y a su vez este produce efectos multiplicadores en el sector servicios. 
El cuadro 1 muestra cómo se distribuyó la población ocupada por rama de actividad económica entre 2005 y 2012, y también presenta la tasa media de crecimiento anual durante este periodo. Se observa también como los sectores de mayor crecimiento son los relacionados al sector servicios y de manera muy relevante la industria extractiva y de la electricidad, el único sector que presenta un crecimiento negativo es el de la industria de la construcción, sector que desde la crisis inmobiliaria en el año 2008 y 2009 en Estados Unidos ha presentado serios problemas en su actividad. Los sectores de mayor dinamismo han sido los vinculados con la industria de transformación, así como los servicios profesionales y financieros, y el sector comercio, éstos dos últimos se caracterizan por contar con una gran cantidad de empleo en actividades informales.

En México la ocupación en el sector informal es una característica del mercado de trabajo, este fenómeno se ha incrementado en la última década, por tal motivo, se considera en este trabajo de investigación que dicha cualidad, pudiera tener diferencias en la estimación de la desigualdad salarial.

Cuadro 1. PEA por rama de actividad económica y tasa de crecimiento medio de cada sector en la Frontera Norte de México, 2005 y 2012

\begin{tabular}{|c|c|c|c|c|c|}
\hline \multirow{2}{*}{$\begin{array}{l}\text { Rama de actividad } \\
\text { económica }\end{array}$} & \multicolumn{2}{|c|}{2005} & \multicolumn{2}{|c|}{2012} & \multirow{2}{*}{$\begin{array}{l}\text { Tasa de } \\
\text { crecimiento }\end{array}$} \\
\hline & Total & $\%$ & Total & $\%$ & \\
\hline $\begin{array}{l}\text { Industria extractiva y de la } \\
\text { electricidad }\end{array}$ & 82511 & 1.2 & 112510 & 1.4 & 4.53 \\
\hline Industria de la transformación & 1658289 & 24.0 & 1772459 & 22.0 & 0.96 \\
\hline Construcción & 671802 & 9.8 & 643519 & 8.1 & -0.61 \\
\hline Comercio & 1463048 & 21.0 & 1729576 & 22.0 & 2.42 \\
\hline $\begin{array}{l}\text { Restaurantes y servicios de } \\
\text { alojamiento }\end{array}$ & 438323 & 6.4 & 550575 & 6.9 & 3.31 \\
\hline $\begin{array}{l}\text { Transportes comunicaciones, } \\
\text { correo y almacenamiento }\end{array}$ & 381185 & 5.6 & 427396 & 5.1 & 1.65 \\
\hline $\begin{array}{l}\text { Servicios profesionales, } \\
\text { financieros y corporativos }\end{array}$ & 1162330 & 17.0 & 1480117 & 19.0 & 3.51 \\
\hline Servicios diversos & 692964 & 10.4 & 913983 & 11.0 & 4.03 \\
\hline $\begin{array}{l}\text { Gobierno y organismos } \\
\text { internacionales }\end{array}$ & 314609 & 4.6 & 360055 & 4.5 & 1.95 \\
\hline Total & 6865061 & 100.0 & 7990191 & 100.0 & 2.19 \\
\hline
\end{tabular}

Fuente: elaboración propia con base en las ENOE, años respectivos. 
Gráfica 2. Tasa de ocupación en el sector informal en la FNM y México, 2005 y 2012

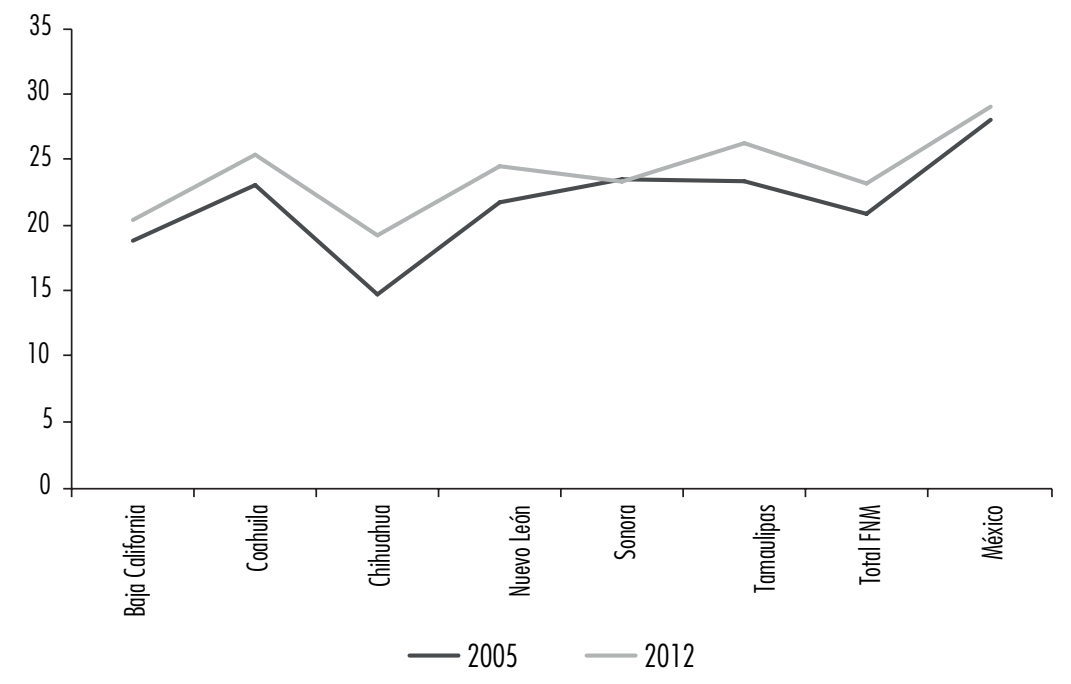

Fuente: Elaboración propia con datos de ENOE, años respectivos.

Para mostrar un panorama generalizado de la tendencia que ha seguido la informalidad en la FNM y en México se presenta la gráfica 2 en la que se muestran las tasas de ocupación en el sector informal para los ańos 2005 y 2012, se observa que los valores para cada entidad son superiores en el ańo 2012, las mayores tasas de participación en dicho sector, las tiene el estado de Tamaulipas y el promedio nacional es superior al promedio de la FNM.

\section{METODOLOGÍA DE LA DESCOMPOSICIÓN EXACTA DE SALARIOS CON EL ÍNDICE DE GINI}

Se utiliza para la aplicación empírica el índice de Gini ${ }^{2}$ debido a la bondad en su interpretación, dicho índice permite comprender de manera sencilla los niveles de desigualdad al estar acotado entre valores de cero a uno (cero con nula desigualdad y uno para el máximo valor asignado). Además su descomto de manera exacta; mediante la fórmula generalizada Shapley es posible tener una nueva fórmula del índice de Gini, con la cual la descomposición resulta ser exacta mostrando la contribución de cada subgrupo al total de la desigualdad, sin que se presente un residuo. 
posición resulta ser una estimación novedosa ya que es posible realizarla de forma exacta.

Para descomponer el índice de Gini, procedemos con la metodología planteada originalmente en Shorrocks (2013), la que permite descomponer de manera exacta (es decir, sin residuos ni componentes aleatorios) cualquier índice de desigualdad utilizando el enfoque teórico de Shapley (1954). La naturaleza particular de esta técnica es que la descomposición es siempre "exacta" en la que los subgrupos considerados pueden ser tratados simétricamente (Shorrocks, 2013: 20; Kolenikov y Shorrocks, 2005). Algunas de las aplicaciones en las que se ha utilizado esta técnica a nivel internacional, han sido para analizar la pobreza y la desigualdad en Rusia (Kolenikov y Shorrocks, 2005), en China (Wan, Lu y Chen, 2007; Wan, 2002; Wan y Zhou, 2005) y en Camerún (Abdelkrim, 2006). Con respecto al caso mexicano ha sido aplicada en los trabajos de Ochoa, Huesca, Calderón (2015) y Rodríguez, Huesca y Camberos (2011) con distintas categorías de trabajo.

Se parte de considerar la ecuación del índice de Gini de Morduch y Sicular (2002), donde se realiza un ordenamiento creciente en los ingresos de los trabajadores como $y_{1} \leq y_{2} \leq \cdots \leq y_{i}$, y se escribe de la siguiente manera:

$G=\frac{2}{n^{2} \mu} \sum_{i=1}^{n}\left(i=\frac{n+1}{2}\right) y_{i}$

La descomposición se calcula como el valor de la desigualdad para cada subgrupo de $k$ trabajadores de la siguiente forma:

$$
S_{\text {Gini }}^{k}=\left(\frac{\sum_{i=1}^{n}\left(i=\frac{n+1}{2}\right) y_{i}^{k}}{\sum_{i=1}^{n}\left(i=\frac{n+1}{2}\right) y_{i}}\right)
$$

Donde $S$ representa el subgrupo limitado al tamaño $S \in\{0,1, \ldots, n-1\}$. Cada subgrupo de trabajadores, aporta un ranking al salario $i$ para $y_{i}^{k}$ sobre el total en la distribución para $y_{i}$. Posteriormente, se obtiene el término $M V\left(\sigma^{i}(w), k\right)$ que se refiere al valor marginal de agregar un trabajador $k$ a su grupo. ${ }^{3}$ 
Así los trabajadores son ordenados aleatoriamente por su dispersión de ingresos $(y)$ mediante el símbolo $\sigma^{i}(y)$ como sigue:

$$
\sigma^{i}(y)=\{\underbrace{\sigma^{1}, \sigma^{2}, \cdots, \sigma^{i-1}}_{s}, \underbrace{\sigma^{i+1}, \cdots, \sigma^{n}}_{n-S-1}\}
$$

De tal forma, es factible escribir la fórmula general del valor Shapley (1954) con el i-ésimo orden posible de subgrupos como sigue:

$$
G_{k}=\frac{1}{n !} \sum_{i=1}^{n !} M V\left(\sigma^{i}, g\right)
$$

El término $M V\left(\sigma^{i}, g\right)$ indica el efecto que se presenta en la estimación, cuando se elimina el grupo $g$ por el orden $\sigma^{i}$ en la contribución del conjunto de grupos $S$. Para el caso de seis grupos (nuestro ejercicio se realiza para seis grupos laborales) se descompone la desigualdad de la siguiente manera:

$$
\begin{aligned}
& G_{1}^{s}=0.166\left[\mu-\mu_{6}-\mu_{5}-\mu_{4}-\mu_{3}-\mu_{2}-\mu_{1}\right] \\
& G_{2}^{s}=0.166\left[\mu-\mu_{6}-\mu_{5}-\mu_{4}-\mu_{3}-\mu_{1}-\mu_{2}\right] \\
& G_{3}^{s}=0.166\left[\mu-\mu_{6}-\mu_{5}-\mu_{4}-\mu_{2}-\mu_{1}-\mu_{3}\right] \\
& G_{4}^{s}=0.166\left[\mu-\mu_{6}-\mu_{5}-\mu_{3}-\mu_{2}-\mu_{1}-\mu_{4}\right] \\
& G_{5}^{S}=0.166\left[\mu-\mu_{6}-\mu_{4}-\mu_{3}-\mu_{2}-\mu_{1}-\mu_{5}\right] \\
& G_{6}^{S}=0.166\left[\mu-\mu_{5}-\mu_{4}-\mu_{3}-\mu_{2}-\mu_{1}-\mu_{6}\right]
\end{aligned}
$$

Las ecuaciones previas, conforman la población con medias de salario $\left[\mu_{1}, K, \mu_{6}\right]$ que aíslan el efecto medio del ingreso $\mu$ y del resto de medias en cada subgrupo asignando el peso correspondiente. Así la descomposición se representa con dos componentes entre e intra, $G_{k}$ que es equivalente al cálculo del índice de Gini de las ecuaciones expresadas en (5a) a (5f) con la siguiente fórmula:

$$
G_{k}=G_{\text {entre }}^{S}+G_{\text {intra }}^{S}
$$


Y sus componentes respectivas como,

$$
\begin{aligned}
& {\left[\begin{array}{ccc}
G_{\text {entre }}^{S_{1}}= & 0.166 & {\left[G(y)-G\left(y_{1}\left(\mu / \mu_{1}\right)\right)+G\left(\mu_{1}\right)-G(\mu)\right]} \\
\vdots & \vdots & \vdots \\
G_{\text {entre }}^{S_{k}}= & 0.1666 & {\left[G(y)-G\left(y_{k}\left(\mu / \mu_{g}\right)\right)+G\left(\mu_{g}\right)-G(\mu)\right]}
\end{array}\right]} \\
& {\left[\begin{array}{ccc}
G_{\mathrm{in} r a}^{S_{1}}= & 0.166 & {\left[G(y)-G\left(\mu_{1}\right)+G\left(y\left(\mu / \mu_{1}\right)\right)-G(\mu)\right]} \\
\vdots & \vdots & \vdots \\
G_{\text {int ra }}^{S_{k}}= & 0.1666 & {\left[G(y)-G\left(\mu_{g}\right)+G\left(y\left(\mu / \mu_{g}\right)\right)-G(\mu)\right]}
\end{array}\right]}
\end{aligned}
$$

$G\left(y\left(\mu / \mu_{q}\right)\right)$ Permite normalizar la desigualdad con el propio vector de medias de ingresos para cada grupo, donde el promedio de los salarios de cada subgrupo será igual a su media; y $G\left(\mu_{g}\right)$ corresponde precisamente a la desigualdad media del grupo en cuestión. En la expresión (7) se deduce la parte de la desigualdad existente entre los grupos y en la expresión (8) se obtiene la parte de la desigualdad al interior de los subgrupos.

\section{Datos utilizados para la descomposición del Gini de los salarios}

Las bases de datos utilizadas corresponden a la Encuesta Nacional de Ocupación y Empleo (ENOE) emitidas por el Instituto Nacional de Estadística y Geografía (INEGi) de los años 2005 al 2012.

El criterio de selección de los trabajadores es de aquellos en edad de trabajar en el rango de 16 a 65 años de edad, tanto hombres como mujeres.

La unidad de análisis corresponde a los seis estados de la Frontera Norte de México (Baja California Norte, Sonora, Chihuahua, Coahuila, Nuevo León y Tamaulipas). Los salarios de los trabajadores son captados por la encuesta de forma mensual en pesos corrientes mexicanos, y procedimos a deflactar a precios de 2005 utilizando el índice de precios al consumidor al segundo trimestre de ese año. ${ }^{4}$

4

Se utiliza la información del segundo trimestre del año para tener información homogénea de la clasificación mexicana de ocupaciones, ya que esta sufrió modificaciones a partir del tercer trimestre de 2012. 


\section{¿Por qué realizar el análisis por tareas?}

Como se mencionó anteriormente, una de las formas de analizar el impacto del cambio tecnológico en el mercado de trabajo, es mediante el estudio de los cambios en la mano de obra y las implicaciones salariales a las que se enfrentan los trabajadores de acuerdo a sus habilidades que pueden ser adquiridas mediante la experiencia o bien, se pueden aproximar mediante los ańos de escolaridad, ya que se considera que dependiendo de la escolaridad se tendrán habilidades distintas.

La clasificación más utilizada para analizar los cambios en la demanda es categorizando a los trabajadores en los calificados y los que no son calificados.

En este trabajo de investigación, con el objetivo de profundizar en el análisis de la desigualdad salarial de acuerdo con las habilidades de los trabajadores, se considera una clasificación que permite ampliar la caracterización del trabajo. Siguiendo los criterios de Acemoglu et al. (2011) los trabajadores se clasifican por el tipo de tareas que realizan en: abstractas, rutinarias y de tipo manual. Dicha clasificación es única en su tipo y en nuestro conocimiento, no ha sido utilizada en el caso mexicano para este tipo de cometido.

Las tareas abstractas, requieren de la resolución de problemas, de intuición, persuasión y creatividad. Los trabajadores que desempeñan este tipo de tareas suelen tener altos niveles de educación; las tareas rutinarias son aquellas que pueden ser desarrolladas por las maquinas o siguiendo reglas de programación motivo que provoca que sean de fácil sustitución; mientras que las tareas manuales son actividades que requieren de adaptación situacional, reconocimiento visual e interacción entre personas, regularmente para el desempeño de estas tareas se requieren trabajadores de baja calificación.

Bajo los criterios de análisis anteriores se utilizó la Clasificación Mexicana de Ocupaciones (смо) para realizar el ordenamiento correspondiente. ${ }^{5}$ Esta clasificación tiene su ventaja por dos aspectos: 1) facilita la agrupación con respecto de la categorización de los trabajadores en su ocupación, y 2) permite conocer de manera sencilla y rápida la dinámica del tipo de ocupación a la que nos referimos.

En resumen, se clasificó a los trabajadores en tres grupos de ocupaciones (abstracta, rutinaria y manual), además con la intención de analizar la dualidad del mercado laboral mexicano se estableció como criterio de formalidad laboral el contar o no con servicio médico, por lo tanto, para efectos de la des-

En el anexo 1 se presenta un cuadro con las tareas que son incluidas en cada una de las categorías. 
composición exacta se cuenta con seis categorías para el grupo de ocupaciones (abstracta formal, abstracta informal, rutinaria formal, rutinaria informal, manual formal y manual informal). Con ello consideramos que se profundiza en la dinámica laboral del mercado de trabajo mexicano.

\section{RESULTADOS DE LA DESCOMPOSICIÓN EN LA DESIGUALDAD SALARIAL}

A continuación se muestra la estructura ocupacional de los trabajadores por tipo de tareas, con el objetivo de observar el efecto que tiene el cambio tecnológico en la región mediante esta clasificación propuesta por Acemoglu et al. (2011). El cuadro 2 muestra el cambio ocupacional en los trabajadores por tipo de tareas en los estados de la FNM y México para los ańos 2005 y 2012. Se observa que en cuanto al tipo de tareas abstractas, rutinarias y manuales en la FNM, existe una mayor presencia de trabajo manual. En México, el empleo por tipo de tareas, muestra un aumento gradual de las ocupaciones manuales $(1.28 \%)$, seguidas de las rutinarias $(1.04 \%)$, mientras que las abstractas son apenas perceptibles en crecimiento.

Cuadro 2. Tasas medias de crecimiento de la ocupación por tipo de tareas, 2005-2012

\begin{tabular}{lccc}
\hline Entidad & Abstracta & Rutinaria & Manual \\
\hline Baja California & -1.45 & 0.80 & 1.15 \\
Coahuila & 0.79 & 1.46 & 2.88 \\
Chihuahua & -2.25 & 0.51 & -0.83 \\
Nuevo León & -4.08 & -1.40 & 0.43 \\
Sonora & -1.02 & 2.55 & 0.87 \\
Tamaulipas & 0.19 & 0.40 & 0.27 \\
Total FNM & -1.56 & 0.51 & 0.71 \\
México & -0.04 & 1.04 & 1.28 \\
\hline
\end{tabular}

Fuente: elaboración propia en base a ENOE, años respectivos. 


\section{Análisis de la descomposición exacta del Gini: tareas y habilidades en el sector formal e informal}

Para realizar el cálculo de la descomposición se toma toda la muestra de trabajadores de acuerdo a su clasificación por tareas, pero se ańadió el toque del sector en el que se realizan, sean éstas de manera formal o informal, así se estará en condiciones de controlar esta característica de trabajo que resulta ser un problema estructural de la economía laboral de México.

Primeramente, se muestra la evolución del coeficiente de Gini durante el periodo de estudio en la gráfica 3, donde se comparan sus valores para México y la Frontera Norte. Se observa una mayor desigualdad en el país respecto a la FNM, en ambos caso la tendencia durante el periodo de análisis es decreciente. Es decir, esta gráfica nos otorga evidencia de que la desigualdad salarial en México y la región de la Frontera Norte han mostrado una reducción en el periodo de análisis.

Los resultados del análisis de descomposición por tipo de tarea se muestran en los cuadros 3 y 4, más adelante. El índice global de desigualdad pasó de 0.406 a 0.369 en el año 2012 para la Frontera Norte, y para México su nivel se ubicó en el 0.4. Es decir, se observa una ligera disminución en la desigualdad este hallazgo va en línea con el trabajo reciente de Campos et al. (2014) quienes mencionan que la desigualdad en México se ha reducido. En nuestra descomposición, podemos inferir con una confianza estadística al 95\% que el componente al interior de los colectivos laborales explica la mayor parte de la desigualdad al pasar de 74 a $71 \%$ entre 2005 y 2012. En el análisis del componente intra para la FNM se explica que la mayor contribución a la desigualdad (aun cuando es decreciente) se presenta en trabajadores que realizan tareas manuales en el sector informal con un 18\%, valor que para el 2012 se incrementó a $19.3 \%$.

En las gráficas 4 y 5 se presentan los componentes entre e intra respectivamente para México y la región de la Frontera Norte. ${ }^{6}$ Se observa que el componente entre-grupos no supera el valor del $32 \%$ a lo largo del periodo, por lo tanto, el $68 \%$ restante se atribuye a la parte intra-grupos.

En ambos componentes los valores para el país son superiores a los que se presentan en la FNM, asimismo, se observa que el elemento intra a lo largo del periodo muestra una reducción.

\footnotetext{
Para este cálculo se ejecutó la descomposición para cada año de la encuesta, y con la intención de que la información sea más clara para el lector se resumen los resultados de todo el periodo gráficamente.
} 
Gráfica 3. Desigualdad salarial en la FNM y México, 2005-2012 (Índice de Gini)

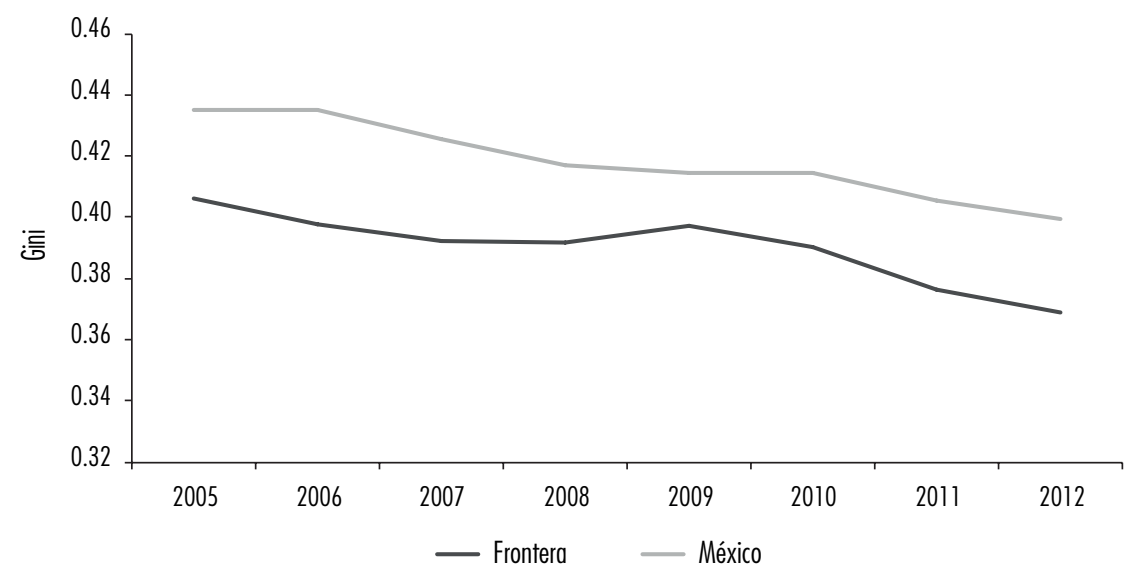

Fuente: estimación propia con datos de ENOE.

Cuadro 3. Descomposición exacta del índice de Gini de la desigualdad salarial por grupos de tareas formales (f) e informales (i), Frontera Norte y México 2005

\begin{tabular}{|c|c|c|c|c|c|c|}
\hline \multirow{2}{*}{$\begin{array}{l}\text { Contribución } \\
\text { y grupo }\end{array}$} & \multicolumn{3}{|c|}{ Frontera } & \multicolumn{3}{|c|}{ México } \\
\hline & Absoluto & Relativo & $\%$ intra & Absoluto & Relativo & $\%$ intra \\
\hline Entre & 0.103 & 0.254 & & 0.130 & 0.298 & \\
\hline Intra & 0.303 & 0.746 & & 0.306 & 0.702 & \\
\hline Gini total & 0.406 & 1.000 & & 0.435 & 1.000 & \\
\hline Subgrupos/Sk & & & $\%$ intra & & & $\%$ intra \\
\hline Ocupaciones & & & & & & \\
\hline Abstracta $f$ & 0.046 & 0.113 & 15.178 & 0.035 & 0.081 & 11.530 \\
\hline Abstracta i & 0.029 & 0.071 & 9.469 & 0.028 & 0.065 & 9.310 \\
\hline Rutinaria $f$ & 0.062 & 0.152 & 20.408 & 0.037 & 0.085 & 12.036 \\
\hline Rutinaria i & 0.058 & 0.143 & 19.156 & 0.077 & 0.177 & 25.194 \\
\hline Manual $\mathrm{f}$ & 0.032 & 0.080 & 10.719 & 0.022 & 0.050 & 7.183 \\
\hline Manual i & 0.076 & 0.187 & 25.070 & 0.106 & 0.244 & 34.747 \\
\hline Suma intra & 0.303 & & 100.000 & 0.306 & & 100.00 \\
\hline
\end{tabular}


Desigualdad salarial y cambio tecnológico en la Frontera Norte de México

\begin{tabular}{|c|c|c|c|c|c|c|}
\hline \multirow{2}{*}{$\begin{array}{l}\text { Contribución } \\
\text { y grupo }\end{array}$} & \multicolumn{3}{|c|}{ Frontera } & \multicolumn{3}{|c|}{ México } \\
\hline & Absoluto & Relativo & $\%$ intra & Absoluto & Relativo & $\%$ intra \\
\hline$G\left(\mu_{g}\right)$ & 0.165 & & & 0.212 & & \\
\hline$G(y(\mu / \mu))$ & 0.365 & & & 0.388 & & \\
\hline
\end{tabular}

Fuente: elaboración propia con base en ENOE 2005.

Cuadro 4. Descomposición exacta del índice de Gini en la desigualdad salarial por grupos de tareas formales (f) e informales (i), Frontera Norte y México 2012

\begin{tabular}{|c|c|c|c|c|c|c|}
\hline \multirow{2}{*}{$\begin{array}{l}\text { Contribución } \\
\text { y grupo }\end{array}$} & \multicolumn{3}{|c|}{ Frontera } & \multicolumn{3}{|c|}{ México } \\
\hline & Absoluto & Relativo & $\%$ intra & Absoluto & Relativo & $\%$ intra \\
\hline Entre & 0.104 & 0.281 & & 0.120 & 0.301 & \\
\hline Intra & 0.265 & 0.719 & & 0.279 & 0.699 & \\
\hline Gini total & 0.369 & 1.000 & & 0.400 & 1.000 & \\
\hline Subgrupos/Sk & & & $\%$ intra & & & $\%$ intra \\
\hline \multicolumn{7}{|l|}{ Ocupaciones } \\
\hline Abstracta f & 0.036 & 0.098 & 13.611 & 0.027 & 0.068 & 9.767 \\
\hline Abstracta i & 0.025 & 0.068 & 9.475 & 0.026 & 0.065 & 9.330 \\
\hline Rutinaria f & 0.053 & 0.143 & 19.901 & 0.032 & 0.080 & 11.404 \\
\hline Rutinaria i & 0.054 & 0.147 & 20.433 & 0.074 & 0.186 & 26.585 \\
\hline Manualf & 0.026 & 0.070 & 9.751 & 0.018 & 0.046 & 6.617 \\
\hline Manual i & 0.071 & 0.193 & 26.829 & 0.101 & 0.254 & 36.297 \\
\hline Suma intra & 0.265 & & 100.000 & 0.279 & & 100.000 \\
\hline$G\left(\mu_{g}\right)$ & 0.169 & & & 0.196 & & \\
\hline$G(y(\mu / \mu))$ & 0.331 & & & 0.355 & & \\
\hline
\end{tabular}

Fuente: elaboración propia con base en ENOE 2012. 
Luis Huesca y Gloria Ochoa

Gráfica 4. Desigualdad salarial entre-grupos por tarea, México y FNM

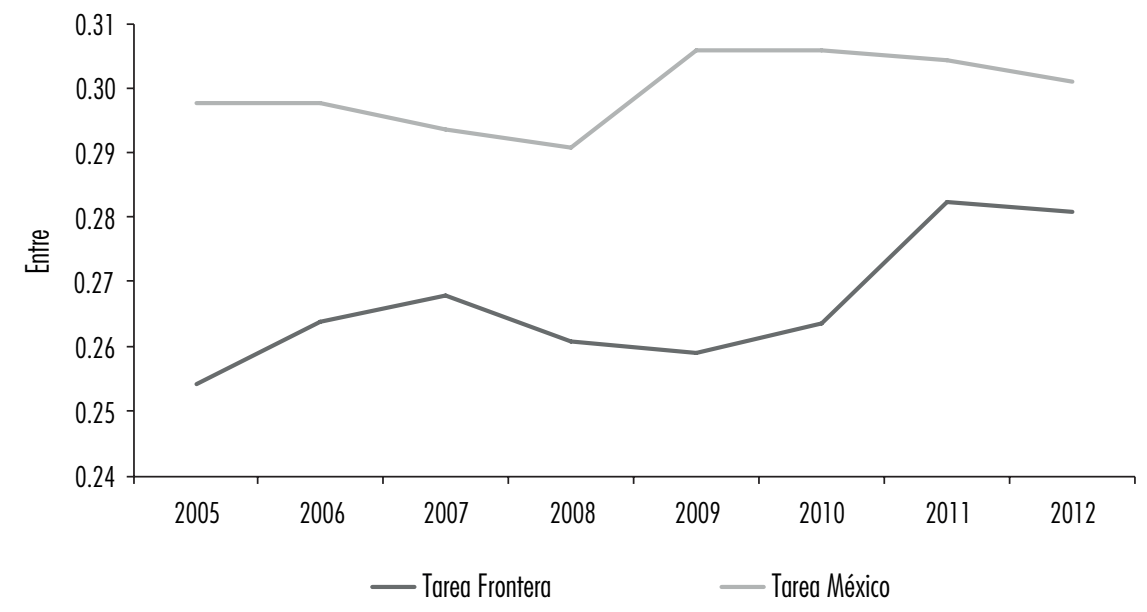

Fuente: elaboración propia con base en las ENOE, años respectivos.

Gráfica 5. Desigualdad salarial intra-grupos por tarea, México y FNM

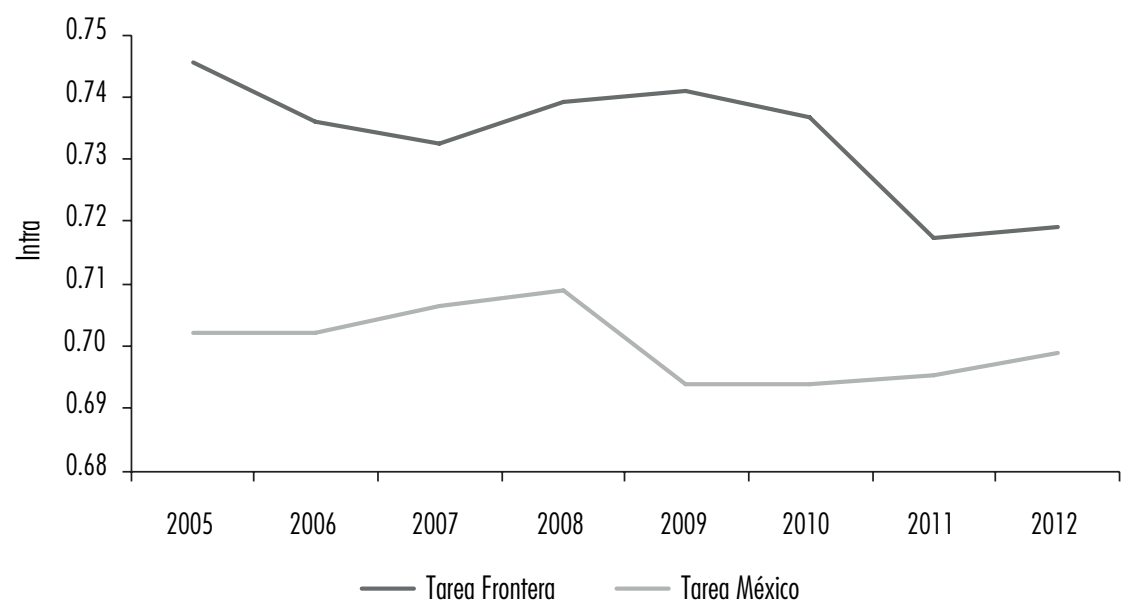

Fuente: elaboración propia con base en las ENOE, años respectivos. 


\section{CONCLUSIONES}

La investigación presenta una aplicación empírica novedosa en el ámbito laboral respecto al análisis de los salarios, que permite explicar la desigualdad ocurrida en México y su región de la Frontera Norte durante los años 20052012 por tipo de tareas y habilidades con las que cuentan los ocupados en el mercado laboral mexicano. El índice Gini se descompone de manera exacta y se muestra con mayor precisión los cambios en la desigualdad salarial.

Los resultados indican que ha sido la parte al interior de los grupos la que ha permitido reducir las distancias de los salarios con una participación del $70 \%$ en el total de la desigualdad; es decir, esta se explica dentro de los mismos grupos de trabajadores y su reducción se observa con el índice de Gini para la FNM que pasó de 0.406 a 0.369 en 2012 y para el país con un valor de 0.435 que se redujo a 0.40 en el último año del análisis.

Una vez que se observó que la desigualdad salarial es explicada al interior de los grupos, es muy importante mostrar la proporción en la que cada uno de estos colectivos de trabajadores aporta a dicha disparidad, en este sentido, los resultados muestran que los trabajadores que realizan tareas manuales en el sector informal aportan un 18\%, valor que para el año 2012 se incrementó a 19.2\% en la región de la Frontera Norte, mientras que para el país el mayor aporte es en el mismo grupo, pero con un porcentaje de participación de $25.4 \%$.

Estos hallazgos permiten concluir que la desigualdad salarial en México se explica en gran medida porque las actividades informales de tipo manual, seguidas de las rutinarias, siendo las abstractas las que menos vínculo tienen con la informalidad y también, las que menos contribuyeron a la reducción en la desigualdad tanto en la FNM como en el país.

Los hallazgos de este trabajo de investigación nos permiten mostrar a la luz de la teoría antes planteada sobre el impacto de la tecnología en el mercado laboral, que de modo contrario a lo sucedido en países como Estados Unidos y Canadá y a lo que sucedió en México en la década de los noventa, donde el cambio tecnológico sesgado provocó un incremento en la desigualdad salarial, en la época reciente en México y su FNM la desigualdad de los salarios se ha reducido, considerándose la principal causa de dicha disminución, la caída de los salarios de los trabajadores más calificados o con mayores grados de escolaridad.

Asimismo, una de las causas que pudiera no hacer diferencias salarial es lo relativo a la introducción de tecnología, misma que pudiera ser insuficiente para generar una demanda con más habilidades o bien, que dichos colectivos pudieran ahora estar realizando actividades de tipo rutinarias. 
Anexo 1. Clasificación en tareas abstractas, rutinarias y manuales

\begin{tabular}{|c|c|}
\hline Abstractas & $\begin{array}{l}\text { - Profesionistas } \\
\text { - Trabajadores en la educación } \\
\text { - Trabajadores en el arte, espectáculos y deportes } \\
\text { - Jefes, supervisores y otros trabajadores de control en la fabricación artesanal } \\
\text { e industrial y en actividades de reparación y mantenimiento } \\
\text { - Jefes de departamento, coordinadores y supervisores en actividades administrativas } \\
\text { - Técnicos }\end{array}$ \\
\hline Rutinarias & $\begin{array}{l}\text { - Trabajadores en apoyo de actividades administrativas } \\
\text { - } \text { Comerciantes, empleados de comercio y agentes de ventas } \\
\text { - } \text { Conductores y ayudantes de conductores de maquinaria móvil y medios de transporte } \\
\text { - } \\
\text { Artesanos y trabajadores fabriles en la industria de la transformación y trabajadores } \\
\text { en actividades de reparación y mantenimiento } \\
\text { - Operadores de maquinaria fija de movimiento continuo y equipos en el proceso de } \\
\text { fabricación industrial }\end{array}$ \\
\hline Manuales & $\begin{array}{l}\text { - Trabajadores en actividades agrícolas, ganaderas, silvícolas, caza y pesca } \\
\text { - Trabajadores en servicios personales en establecimientos } \\
\text { - Trabajadores en servicios domésticos } \\
\text { - Ayudantes, peones y similares en el proceso de fabricación artesanal e industrial en } \\
\text { actividades de reparación y mantenimiento }\end{array}$ \\
\hline
\end{tabular}

Fuente: elaboración propia con información de CMO.

Anexo 2. Estadística descriptiva en desglose de clasificación laboral funcional, México y Frontera Norte (ENOE 2005 y 2012).

Cuadro A1. Estadística descriptiva de la muestra, México ENOE 2005 y 2012 (salario en pesos por semana $2005=100$ )

\begin{tabular}{lrrrrr}
\hline \multicolumn{5}{c}{ Año 2005 } \\
\hline Variables & Media salarios & Desv. Est. & Min. & Max. & $\begin{array}{c}\text { Muestra } \\
\text { expandida }\end{array}$ \\
\hline Por tipo de tareas & & & & & \\
\hline Abstracta formal & 1869.03 & 1541.19 & 44.34 & 59584.30 & 4009508 \\
Abstracta informal & 1605.26 & 2528.98 & 11.55 & 103926.10 & 1988716 \\
Rutinaria formal & 968.76 & 778.96 & 37.64 & 19399.54 & 5105112 \\
Rutinaria informal & 710.08 & 906.37 & 2.31 & 79461.20 & 6880740
\end{tabular}


Desigualdad salarial y cambio tecnológico en la Frontera Norte de México

\begin{tabular}{|c|c|c|c|c|c|}
\hline Manual formal & 947.37 & 630.88 & 34.64 & 24842.26 & 3209410 \\
\hline Manual informal & 654.74 & 938.22 & 3.93 & 173803.00 & 9615709 \\
\hline \multicolumn{6}{|l|}{ Por habilidades } \\
\hline Alta formal & 2196.91 & 1821.46 & 69.28 & 59584.30 & 1571575 \\
\hline Alta informal & 1963.13 & 2149.96 & 13.39 & 34642.03 & 785384 \\
\hline Media formal & 1282.60 & 947.56 & 44.34 & 19861.43 & 4995951 \\
\hline Media informal & 1021.93 & 1972.84 & 7.62 & 173803.00 & 3576394 \\
\hline Baja formal & 897.54 & 627.48 & 34.64 & 34642.03 & 5756504 \\
\hline Baja informal & 673.92 & 915.34 & 2.31 & 103926.10 & 14123387 \\
\hline \multicolumn{6}{|c|}{ Año 2012} \\
\hline \multicolumn{6}{|c|}{ Por tipo de tareas } \\
\hline Abstracta formal & 2194.61 & 1508.00 & 32.79 & 33764.43 & 3801369 \\
\hline Abstracta informal & 1776.02 & 2183.91 & 9.93 & 69284.06 & 2182525 \\
\hline Rutinaria formal & 1250.70 & 1015.87 & 59.58 & 66542.95 & 5133006 \\
\hline Rutinaria informal & 866.56 & 859.09 & 6.93 & 29792.15 & 7688406 \\
\hline Manual formal & 1206.19 & 784.55 & 23.09 & 24826.79 & 3090146 \\
\hline Manual informal & 852.96 & 795.63 & 4.85 & 38491.22 & 10922945 \\
\hline \multicolumn{6}{|l|}{ Por habilidades } \\
\hline Alta formal & 2459.93 & 1757.96 & 98.15 & 26327.95 & 1618061 \\
\hline Alta informal & 2130.51 & 2293.06 & 20.79 & 36951.50 & 809215 \\
\hline Media formal & 1570.50 & 1164.88 & 27.71 & 49657.50 & 5459590 \\
\hline Media informal & 1123.75 & 1383.98 & 11.55 & 69284.06 & 4820612 \\
\hline Baja formal & 1142.75 & 792.42 & 23.09 & 66542.95 & 5010130 \\
\hline Baja informal & 821.94 & 757.44 & 4.85 & 41709.01 & 15184787 \\
\hline
\end{tabular}

Fuente: elaboración propia con base a ENOE 2005 y 2012. 
Cuadro A2. Estadística descriptiva de la muestra, Frontera ENOE 2005 y 2012

(salario en pesos por semana $2005=100$ )

Aก̃o 2005

\begin{tabular}{|c|c|c|c|c|c|}
\hline Variables & Media salarios & Desv. Est. & Min. & Max. & $\begin{array}{c}\text { Muestra } \\
\text { expandida }\end{array}$ \\
\hline \multicolumn{6}{|c|}{ Por tipo de tareas } \\
\hline Abstracta formal & 1938.59 & 1531.99 & 46.19 & 23094.69 & 983437 \\
\hline Abstracta informal & 1939.35 & 2643.75 & 46.19 & 69284.06 & 390848 \\
\hline Rutinaria formal & 1027.09 & 716.13 & 61.89 & 9237.88 & 1647128 \\
\hline Rutinaria informal & 905.65 & 1040.62 & 6.93 & 16166.28 & 905072 \\
\hline Manual formal & 1044.22 & 768.17 & 34.64 & 23849.19 & 891025 \\
\hline Manual informal & 910.30 & 2474.97 & 3.93 & 173803.00 & 1337517 \\
\hline \multicolumn{6}{|l|}{ Por habilidades } \\
\hline Alta formal & 2422.80 & 1902.98 & 105.77 & 23094.69 & 380607 \\
\hline Alta informal & 2540.90 & 2417.91 & 46.19 & 19861.43 & 155455 \\
\hline Media formal & 1387.98 & 1014.72 & 46.19 & 18475.75 & 1319550 \\
\hline Media informal & 1361.85 & 3995.54 & 11.55 & 173803.00 & 584014 \\
\hline Baja formal & 954.12 & 626.31 & 34.64 & 23849.19 & 1821433 \\
\hline Baja informal & 845.13 & 891.63 & 3.93 & 34642.03 & 1893968 \\
\hline
\end{tabular}

Aก̃o 2012

\begin{tabular}{lrrrrr}
\hline Por tipo de tareas & \multicolumn{7}{c}{} \\
\hline Abstracta formal & 2261.66 & 1706.87 & 32.79 & 33764.43 & 850646 \\
Abstracta informal & 1898.77 & 2281.39 & 57.74 & 36951.50 & 380684 \\
Rutinaria formal & 1271.72 & 840.72 & 64.43 & 19907.62 & 1602776 \\
Rutinaria informal & 907.10 & 894.84 & 21.71 & 12413.39 & 1040581 \\
Manual formal & 1212.44 & 691.39 & 124.71 & 9931.41 & 843635 \\
Manual informal & 937.53 & 1016.65 & 9.47 & 38491.22 & 1498057 \\
\hline Por habilidades & & & & & \\
\hline Alta formal & 2712.60 & 1895.97 & 138.57 & 16166.28 & 367145 \\
Alta informal & 2478.60 & 3165.14 & 57.74 & 36951.50 & 138162
\end{tabular}




\begin{tabular}{lrrrrr} 
Media formal & 1585.20 & 1156.45 & 32.79 & 33764.43 & 1313970 \\
Media informal & 1212.51 & 1240.82 & 27.71 & 14896.07 & 719918 \\
Baja formal & 1157.81 & 679.41 & 64.43 & 19907.62 & 1622057 \\
Baja informal & 894.62 & 945.52 & 9.47 & 38491.22 & 2063284 \\
\hline
\end{tabular}

Fuente: elaboración propia con base a ENOE 2005 y 2012.

\section{BIBLIOGRAFÍA}

Abdelkrim, Araar (2006), "On the Decomposition of the Gini Coefficient: An Excact Approach, with an Illustration Using Cameroonian Data", $C a-$ hier de Recherche, Working Paper 06-02.

Acemoglu, Daron and David Autor (2011), "Skills, Tasks and Technologies: Implications for Employment and Earnings", Handbook of Labor Economics, 4B, pp. 1043-1171.

Autor, David (2013), "The 'Task Approach' to Labor Markets: An Overview" (No. w18711), National Bureau of Economic Research, Working Paper 1320, Massachusetts, MIT.

Autor, David, Lawrence Katz and Melissa Kearney (2008), "Trends in U.S. Wage Inequality: Revising the Revisionists", The Review of Economic and Statistics, XC(2), pp. 300-323.

Ayala, Edgar, Joana Chapa e Izabel Hernández (2009), "Integración regional binacional: Evidencia para los estados del Norte de México y Texas", Frontera Norte, 21(41), pp. 105-123.

Campos, Raymundo, Gerardo Esquivel and Nora Lustig (2014), "The Rise and Fall of income Inequality in Mexico, 1989-2010”, Wider Working Paper, núm. 2012, pp. 140-163.

Castro, David (2007), "Disparidad salarial urbana en México, 1992-2002", Estudios Sociales, 29, pp. 117-153.

Castro, David y Luis Huesca (2007), "Desigualdad salarial en México: una revisión”, Papeles de Población, 54, pp. 225-264.

Esquivel, Gerardo and José Rodriguez (2003), "Technology, Trade, and Wage Inequality in Mexico before and after NAFTA", Journal of Development Economics, 72, pp. 543-565.

Hanson, Gordon (2003), "What has happened to Wages in Mexico since NAFTA? Implications for Hemispheric Free Trade", Working Paper Series, núm. 9563, pp. 1-43. 
Huesca, Luis y Reyna Rodríguez (2008), "Salarios y calificación laboral en México", Problemas del Desarrollo, Revista Latinoamericana de Economia, 39(154), pp. 61-86.

Kolenikov, Stanislav y Anthony Shorrocks (2005), "A Decomposition Analysis of Regional Poverty in Russia”, Review of Development Economics, 9(1), pp. 25-46.

Lustig, Nora, Luis López and Eduardo Ortiz (2013), "Deconstructing the Decline in Inequality in Latin America", Policy Research Working Paper, núm. 6552.

Mendoza, Eduardo (2010), "El mercado laboral en la Frontera Norte de México: estructura y políticas de empleo", Estudios Fronterizos, 21, pp. $9-42$.

Morduch, Jonathan y Terry Sicular (2002), "Rethinking Inequality Decomposition, with Evidence from Rural China”, Economic Journal, 112 (476), pp. 93-106.

Ochoa, Gloria, Luis Huesca y Cuauhtémoc Calderón (2015), "Descomposición de la desigualdad salarial en los estados de la Frontera Norte de México", Economía Informa, 393, pp. 3-20.

Rodríguez, Reyna, Luis Huesca y Mario Camberos (2011), "Mercado laboral, inequidad salarial y cambio tecnológico", Frontera Norte, núm. 45, Tijuana, México El Colegio de la Frontera Norte, enero-junio, pp. 7-33.

Shapley, Lloy (1954), "A Value for N-person Games", en Harold Khun y Albert Tucker (eds.), Contributions to the Theory of Games 2, Princeton, NJ, Princeton University Press, pp. 307-318.

Shorrocks, Anthony (2013), "Decomposition Procedures for Distributional Analysis: a Unified Framework Based on the Shapley Value", Journal of Economic Inequality, pp. 1-28.

(1984), "Inequality Decomposition by Population Subgroup", Econométrica, 52 (6), pp. 1369-1385.

(1982), "Inequality Decomposition by Factor Components", Econométrica, 50(1), pp. 193-211.

Wan, Guang (2002), "Regression-based Inequality Decomposition. Pitfalls and a Solution Procedure", Discussion Paper, núm. 2002/101, pp. 1-12.

Wan, Guang, Lu, Min y Chen, Zhao (2007), "Globalization and Regional Income Inequality: Empirical Evidence from within China", Review of Income and Wealth, 53(1), pp. 35-59.

Wan, Guanghua y Zhou, Zhangyue (2005), "Income Inequality in Rural China: Regression Based Decomposition Using Household Data", Review of Development Economics, 9(1), pp. 107-120. 